\title{
Comparative Prognostic and Diagnostic Value of Myocardial Blood Flow and Myocardial Flow Reserve After Cardiac Transplantation
}

\author{
Robert J.H. Miller*1, Osamu Manabe*1,2, Balaji Tamarappoo ${ }^{1}$, Sean Hayes ${ }^{1}$, John D. Friedman ${ }^{1}$, Piotr J. Slomka ${ }^{1}$, \\ Jignesh Patel ${ }^{3}$, Jon A. Kobashigawa ${ }^{3}$, and Daniel S. Berman ${ }^{1}$ \\ ${ }^{I}$ Departments of Imaging and Medicine, Cedars-Sinai Medical Center, Los Angeles, California; ${ }^{2}$ Department of Nuclear Medicine, \\ Hokkaido University Graduate School of Medicine, Sapporo, Japan; and ${ }^{3}$ Smidt Heart Institute, Cedars-Sinai Heart Institute, \\ Cedars-Sinai Medical Center, Los Angeles, California
}

\begin{abstract}
Cardiac allograft vasculopathy (CAV) is a major cause of graft failure after cardiac transplantation. CAV is characterized by diffuse involvement of epicardial coronary arteries and the microvasculature. PET allows quantification of absolute myocardial blood flow (MBF) and myocardial flow reserve (MFR), which may be accurate markers of CAV severity. We compared the diagnostic and prognostic utility of stress MBF and MFR after cardiac transplantation. Methods: This was a cohort study of consecutive cardiac transplant patients undergoing ${ }^{82} \mathrm{Rb} \mathrm{PET}$ scans. Semiquantitative regional analysis and global measurement of stress MBF and MFR were performed. Associations with all-cause mortality were assessed with multivariable Cox analysis. The diagnostic accuracy for significant CAV (grade $2 / 3$ ) and the prognostic accuracy of stress MBF and MFR, corrected and uncorrected for rate-pressure product, were compared. Results: In total, 99 patients, mean age 68.8 y and $75.8 \%$ male, were followed for a median of $3.4 \mathrm{y}$, during which 26 deaths occurred. Stress MBF and MFR had similar diagnostic accuracy for significant CAV. However, uncorrected MFR had improved discrimination for all-cause mortality compared with stress MBF (area under the curve, 0.748 vs. 0.639; $P=0.048$ ). Higher MFR (adjusted hazard ratio, 0.30; $P<0.001$ ), but not stress MBF (adjusted hazard ratio, $1.14 ; P=0.656$ ), was associated with reduced all-cause mortality. Preserved MFR $(>2.0)$ identified relatively low-risk patients (annual mortality, $4.7 \%$ ), whereas the presence of a left ventricular ejection fraction lower than $45 \%$ and MFR lower than 1.7 identified high-risk patients (annual mortality, 51.6\%). Conclusion: Quantitative PET analysis, and particularly MFR, has diagnostic and prognostic utility after heart transplantation. Preserved MFR identifies low-risk patients, whereas the presence of multiple abnormal parameters identifies high-risk patients.
\end{abstract}

Key Words: heart transplantation; myocardial flow reserve; myocardial blood flow; PET

J Nucl Med 2020; 61:249-255

DOI: 10.2967/jnumed.119.229625

Received Apr. 9, 2019; revision accepted Jul. 15, 2019.

For correspondence contact: Daniel S. Berman, Cedars-Sinai Medical Center, 8700 Beverly Blvd., Los Angeles, CA 90048.

E-mail: bermand@cshs.org

${ }^{*}$ Contributed equally to this work.

Published online Aug. 26, 2019.

COPYRIGHT (C 2020 by the Society of Nuclear Medicine and Molecular Imaging.
H eart transplantation is a definitive therapy for patients with end-stage heart failure. Because of advances in posttransplant patient care, median posttransplant survival is now over 13 y (1). As long-term survival has increased, the prevalence of cardiac allograft vasculopathy (CAV), characterized by a diffuse arteriopathy involving the epicardial coronary arteries and microvasculature, has increased (2). CAV accounts for over a third of deaths in patients who survive at least $5 \mathrm{y}$ after receiving the transplant and is the most common indication for retransplantation in patients who survive 1 y (3).

Although invasive coronary angiography and intravascular ultrasound have been the gold standard for diagnosing CAV, they are associated with inherent procedural risk (4). Noninvasive monitoring of CAV may provide valuable diagnostic information while avoiding the risk of invasive studies (2). Noninvasive imaging with stress echocardiography and SPECT have poor sensitivity for diagnosis of CAV $(5,6)$. However, PET myocardial perfusion imaging (MPI) offers the advantage of measurement of absolute myocardial blood flow (MBF) and calculation of myocardial flow reserve (MFR). Stress MBF and MFR provide physiologic assessments of both epicardial coronary artery and coronary microvascular function (7). Thus, PET may provide a more comprehensive assessment for CAV than other noninvasive modalities. In previous studies, both MBF and MFR have provided incremental diagnostic and prognostic utility in the semiquantitative assessment of perfusion for the diagnosis of CAV after cardiac transplantation $(8-10)$.

Although evidence regarding the use of PET for CAV surveillance has grown, it has not been adopted as standard clinical practice. Previous studies have used variable thresholds of MBF and MFR for diagnosis of CAV. Additionally, there is no consensus on whether MFR or stress MBF should be used as the marker for adverse cardiovascular outcomes. Our objectives were to compare the diagnostic value of MBF and MFR for detecting CAV, to evaluate the prognostic utility of MBF and MFR for predicting adverse outcomes, and to evaluate the performance of previously described thresholds for ${ }^{82} \mathrm{Rb}$ PET MPI parameters in patients after cardiac transplantation.

\section{MATERIALS AND METHODS}

\section{Study Population}

In total, 105 consecutive patients who underwent pharmacologic stress ${ }^{82} \mathrm{Rb}$ PET after cardiac transplantation between April 2010 and 
December 2015 were identified at a single center. Patients without dynamic data were excluded $(n=6)$. In patients with multiple studies, the first study with dynamic data was included. This study was approved by the Institutional Review Board at Cedars-Sinai Medical Center (CR00013886), and written informed consent was obtained from all patients.

Transplant demographics were obtained from medical records. A history of acute cellular rejection was defined as biopsy showing cellular rejection of at least grade $2 \mathrm{R}$ or a history of treated acute cellular rejection. A history of antibody-mediated rejection was defined as a history of treated antibody-mediated rejection. The invasive coronary angiography examination that most closely preceded PET was reviewed and graded for CAV according to the International Society of Heart and Lung Transplantation classification based on the interpretation of the performing cardiologist, masked to the PET results (11).

\section{Image Acquisition and Reconstruction}

Patients were imaged with a whole-body PET/CT scanner (Siemens Biograph-64 TruePoint PET/CT with the True V) (12). A 6-min listmode rest acquisition was performed simultaneously with injection of 925-1,850 MBq of ${ }^{82} \mathrm{Rb}$. Regadenoson or adenosine stress acquisitions were then performed with the same protocol (13). Heart rate and blood pressure were recorded before ${ }^{82} \mathrm{Rb}$ injection and at peak stress. The 6-min rest and stress data were reconstructed into a dynamic imaging series consisting of 16 frames $(12 \times 10 \mathrm{~s}, 2 \times 30 \mathrm{~s}, 1 \times$ $60 \mathrm{~s}$, and $1 \times 120 \mathrm{~s}$ ) using the vendor iterative method (Fourier rebinning +2 -dimensional attenuation-weighted ordered-subsets expectation maximization) with 2 iterations, 8 subsets, and an $8-\mathrm{mm}$ gaussian postprocessing filter (14). CT attenuation-correction scans were acquired both before rest imaging and after stress imaging. Registration of the CT attenuation map with the PET images was verified visually by an experienced technologist.

Perfusion was assessed semiquantitatively at stress and rest to derive the summed stress score (SSS), summed rest score (SRS), and summed difference score (SDS) (15). Patients were grouped according to abnormalities: SRS ( $\geq 1$ or 0 ), 2 criteria for SSS (SSS $\geq 4$ or $<4$ and $>1$ or $\leq 1$ ), and ischemia (SDS threshold $>1$ ). Left ventricular ejection fraction (LVEF) was assessed at rest. For quantitative measurements, MBF was computed from the dynamic imaging series with dedicated software (QPET; Cedars-Sinai Medical Center) $(14,16)$. A standard 1-tissue-compartment model and ${ }^{82} \mathrm{Rb}$ extraction fraction derived from the described Renkin-Crone function was used to estimate MBF from $K_{1}(17)$. MFR was calculated as MBF at stress divided by $\mathrm{MBF}$ at rest. Corrected rest $\mathrm{MBF}$ was calculated as MBF at rest $\times$ rate-pressure product $/ 10,000$, and corrected MFR was calculated as stress MBF divided by corrected MFR. Rate-pressure product was calculated as heart rate $\times$ systolic blood pressure.

Thresholds for quantitative PET analysis were based on previously published values (MFR $<2.0(8)$ or $<1.75(9)$; stress MBF $<3.7(8)$ or $<1.7(10)$ ). Additionally, we investigated combinations of parameters previously described as having prognostic value ( $S S S \geq 4, \mathrm{LVEF} \leq$ 45 , or MFR $<1.75$ (9); and MBF $<1.7$ with SSS $>1$ or $\mathrm{LVEF} \leq 45$

TABLE 1

Baseline Population Characteristics

\begin{tabular}{|c|c|c|c|}
\hline Characteristic & No death $(n=73)$ & Death $(n=26)$ & $P$ \\
\hline Age (y) & $66.7 \pm 10.5$ & $74.0 \pm 7.3$ & 0.001 \\
\hline Male & $54(74.0 \%)$ & $21(80.8 \%)$ & 0.599 \\
\hline Age at transplantation (y) & $54.3 \pm 11.1$ & $61.9 \pm 6.5$ & 0.001 \\
\hline Donor age $(y)$ & $30.2 \pm 11.9$ & $35.4 \pm 10.7$ & 0.089 \\
\hline Time after transplantation $(\mathrm{y})$ & $12.5 \pm 5.2$ & $12.5 \pm 5.4$ & 0.977 \\
\hline Body mass index $\left(\mathrm{kg} / \mathrm{m}^{2}\right)$ & $26.5 \pm 5.6$ & $25.8 \pm 5.0$ & 0.560 \\
\hline Hypertension & $62(84.9 \%)$ & $19(73.1 \%)$ & 0.236 \\
\hline Diabetes & $31(42.5 \%)$ & $15(57.7 \%)$ & 0.252 \\
\hline Dyslipidemia & $53(72.6 \%)$ & $21(80.8 \%)$ & 0.600 \\
\hline Former smoker & $4(5.5 \%)$ & $2(7.7 \%)$ & 0.651 \\
\hline Renal failure & 7 (9.6\%) & $4(15.4 \%)$ & 0.472 \\
\hline CAV grade* $(0 / 1 / 2 / 3)$ & $46 / 17 / 5 / 3$ & $13 / 6 / 2 / 4$ & 0.489 \\
\hline Cytomegalovirus viremia & $10(13.7 \%)$ & $3(11.5 \%)$ & 1.000 \\
\hline History of acute cellular rejection & $10(13.7 \%)$ & $5(19.2 \%)$ & 0.531 \\
\hline History of antibody-mediated rejection & $4(5.5 \%)$ & $4(15.4 \%)$ & 0.154 \\
\hline \multicolumn{4}{|l|}{ Medication use } \\
\hline Aspirin & $39(53.4 \%)$ & $16(61.5 \%)$ & 0.501 \\
\hline$\beta$-blockers & $32(43.8 \%)$ & $9(34.6 \%)$ & 0.490 \\
\hline Angiotensin-converting inhibitor or receptor blocker & $36(49.3 \%)$ & $11(42.3 \%)$ & 0.649 \\
\hline Diuretics & $16(21.9 \%)$ & $9(34.6 \%)$ & 0.292 \\
\hline Statins & $58(79.5 \%)$ & $16(61.5 \%)$ & 0.113 \\
\hline Calcineurin inhibitor & $63(86.3 \%)$ & $22(84.6 \%)$ & 1.000 \\
\hline Mammalian target of rapamycin inhibitor & $32(43.8 \%)$ & $9(34.6 \%)$ & 0.490 \\
\hline
\end{tabular}

*No death $(n=71)$; death $(n=25)$.

Data are expressed as number followed by percentage in parentheses or as mean \pm SD. 
TABLE 2

Imaging Characteristics

\begin{tabular}{|c|c|c|c|}
\hline Characteristic & No death $(n=73)$ & Death $(n=26)$ & $P$ \\
\hline Resting heart rate & $81.88 \pm 12.5$ & $81.5 \pm 12.8$ & 0.980 \\
\hline Rate-pressure product $(\mathrm{bpm} \times \mathrm{mm} \mathrm{Hg})$ & $10,895 \pm 2,229$ & $11,122 \pm 1,627$ & 0.635 \\
\hline Resting LVEF (\%) & $65.4 \pm 9.7$ & $56.8 \pm 13.1$ & $<0.001$ \\
\hline \multicolumn{4}{|l|}{${ }^{82} \mathrm{Rb}$ semiquantitative imaging } \\
\hline SRS & $0(0-0)$ & $0(0-1)$ & 0.136 \\
\hline SSS & $0(0-2)$ & $0(0-8)$ & 0.102 \\
\hline SDS & $0(0-1)$ & $0(0-4)$ & 0.072 \\
\hline \multicolumn{4}{|l|}{${ }^{82} \mathrm{Rb}$ quantitative imaging } \\
\hline Rest total perfusion deficit & $0(0-0.3)$ & $0.2(0.0-1.5)$ & 0.018 \\
\hline Stress total perfusion deficit & $1.1(0.0-4.4)$ & $2.1(0.6-7.9)$ & 0.111 \\
\hline Ischemic total perfusion deficit & $1.1(0.0-3.9)$ & $1.9(0.5-5.8)$ & 0.200 \\
\hline Rest MBF (mL/min/g) & $1.29(1.06-1.44)$ & $1.29(1.14-1.56)$ & 0.216 \\
\hline Stress MBF $(\mathrm{mL} / \mathrm{min} / \mathrm{g})$ & $2.88(2.41-3.60)$ & $2.54(1.71-3.24)$ & 0.024 \\
\hline MFR & $2.37(2.01-2.80)$ & $1.69(1.28-2.19)$ & $<0.001$ \\
\hline
\end{tabular}

Data are expressed as mean \pm SD or as median followed by IQR in parentheses.

(10)). Additionally, cutoffs were derived from the receiver-operatingcharacteristic curves generated in this study using the Youden index (18).

\section{Outcomes}

The diagnostic outcome was the presence of CAV grade 2 or 3 . The primary prognostic outcome was all-cause mortality. Cardiovascular mortality, the secondary prognostic outcome, was determined after review of all available clinical information based on standard definitions (19). No patients in our cohort underwent retransplantation. Follow-up of all patients, including patients who transferred to alternate transplant programs, was confirmed by trained research coordinators.

\section{Statistical Analyses}

Continuous variables with normal distributions are presented as mean $\pm \mathrm{SD}$ and compared with a Student $t$ test. Continuous variables that were not normally distributed are presented as median and interquartile range (IQR) and compared using the Mann-Whitney $U$ test. Categoric variables are summarized as number and proportion and compared using a Fisher exact test.

The primary outcome was assessed using Kaplan-Meier survival curves and compared using the log-rank test. Univariable and multivariable Cox proportional hazards analyses were performed to identify associations with all-cause mortality and cardiovascular mortality. Multivariable models included variables identified as significant on univariable analyses $(P<0.05)$.

Receiver-operating-characteristic curves were generated for semiquantitative and quantitative PET parameters. Area under the curve (AUC) was compared using the method established by DeLong et al. (20). Calibration of quantitative PET measurements was assessed using the Hosmer-Lemeshow goodness-of-fit test and was found to be adequate for each combination of measurement and outcome (all $P>0.05$ ). Event rates were extrapolated from Cox proportional hazards models. The proportional hazards assumption was assessed with Schoenfeld residuals for each model and found to be valid. We assessed interactions between each of the variables in the final model, with no significant interactions identified. All statistical tests were 2-sided, with a $P$ value of less than 0.05 considered significant. Analysis was performed using JMP (version 13; SAS) and Stata (version 13; StataCorp).

\section{RESULTS}

\section{Follow-up and All-Cause Mortality}

In total, 99 patients were included. Their baseline characteristics are outlined in Table 1 . The mean age was $68.8 \pm 10.0 \mathrm{y}$, and $75(75.8 \%)$ patients were male. Median resting heart rate was 82 (IQR, 73-90). During a median follow-up of 3.4 y (IQR, 2.2-4.0 y), 26 patients died $(26.3 \%)$. The patients who died were older at the time of PET (mean age, 74.0 vs. 66.7; $P<0.001$ ) and had a lower mean LVEF (56.8\% vs. $65.4 \%, P<0.001)$.

Imaging characteristics are outlined in Table 2 . There were no significant differences in SSS, SRS, or SDS between patients with and without all-cause mortality. However, stress MBF (2.54 vs. $2.88 \mathrm{~mL} / \mathrm{min} / \mathrm{g}, P=0.024)$ and MFR (1.69 vs. $2.37, P<0.001)$ were lower in patients who died during follow-up.

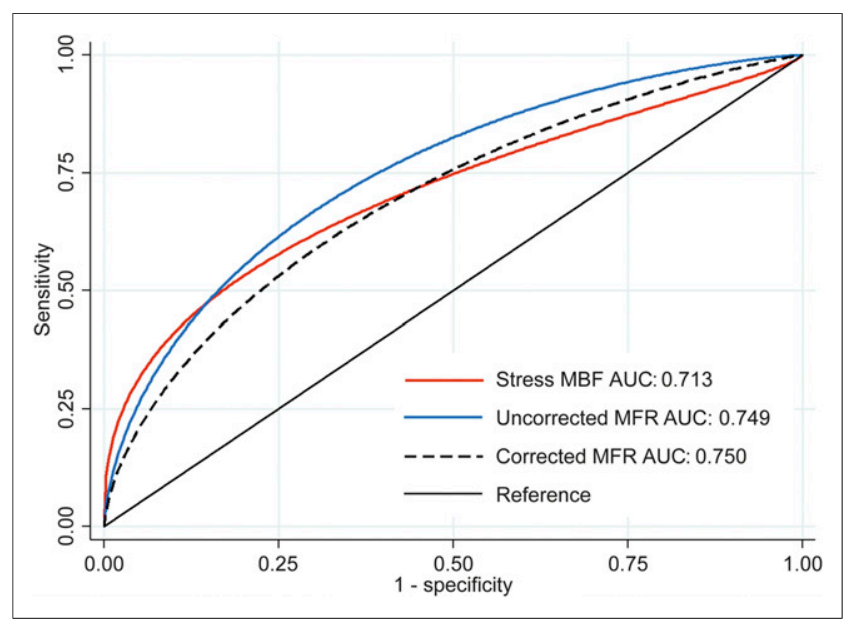

FIGURE 1. Receiver-operating-characteristic curves for diagnosing $\mathrm{CAV} \geq$ grade 2 . There was no difference between uncorrected MFR AUC and stress MBF AUC $(P=0.499)$ or corrected MFR AUC $(P=0.310)$. 


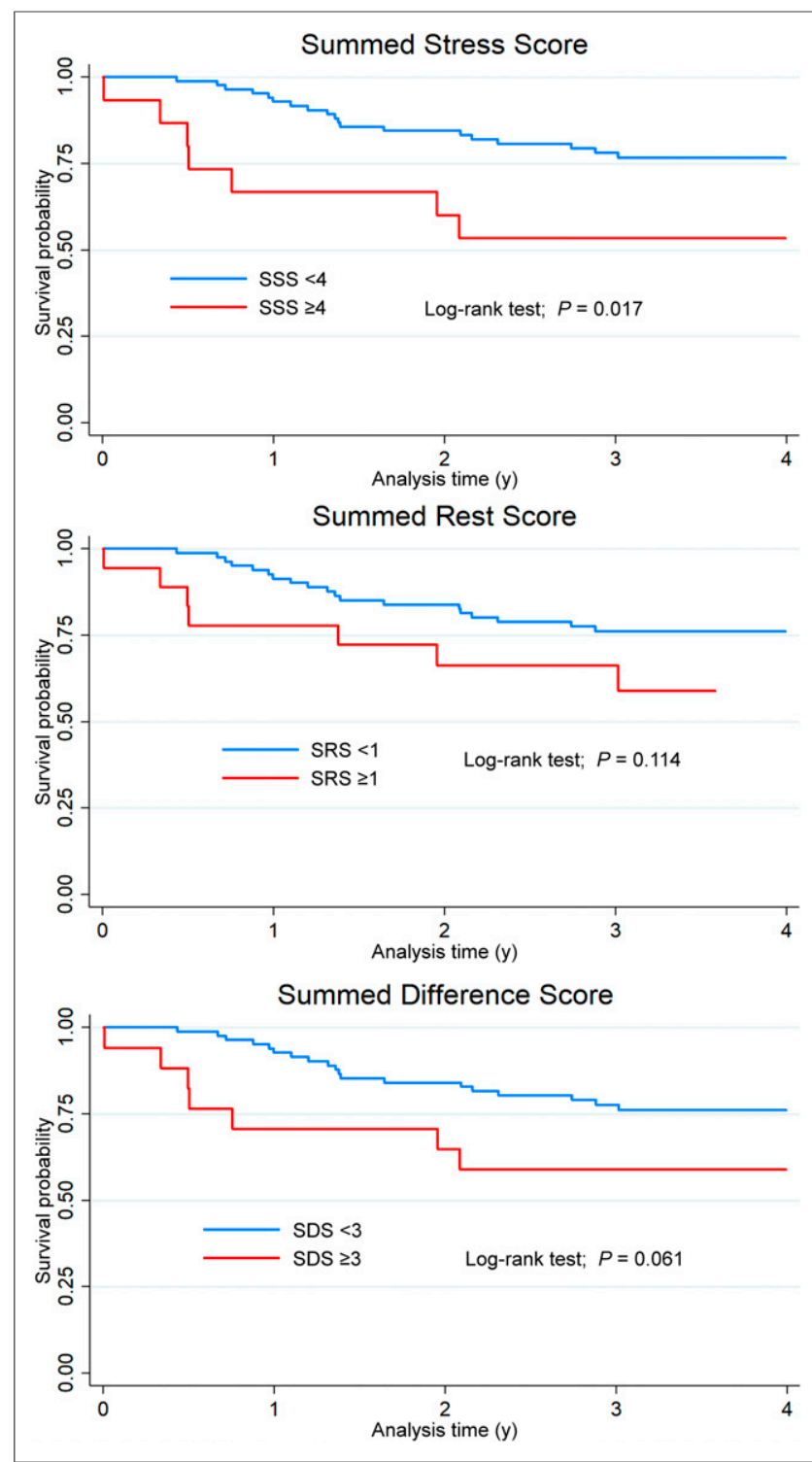

FIGURE 2. Kaplan-Meier survival curves for all-cause mortality stratified by presence of abnormal regional perfusion. Patients with $S S S \geq 4$ were more likely to experience all-cause mortality during follow-up (logrank $P=0.017$ ).

\section{Diagnostic Utility of ${ }^{82} \mathrm{Rb}$ PET}

Reference angiography occurred at a median of $1.0 \mathrm{y}$ (IQR, 0.9$2.0 \mathrm{y}$ ) before the PET study. MBF, MFR, and corrected MFR demonstrated good discrimination of significant CAV. There were no significant differences in the ability of stress MBF (AUC, 0.713), MFR (AUC, 0.749), or corrected MFR (AUC, 0.714) to identify patients with significant CAV (Fig. 1). Optimal cutoffs in our population were as follows: a stress MBF of less than 2.83 (sensitivity, $73.1 \%$; specificity, 56.2\%), an uncorrected MFR of less than 2.22 (sensitivity, $80.8 \%$; specificity, $61.6 \%$ ), and an corrected MFR of less than 2.19 (sensitivity, $76.9 \%$; specificity, $65.8 \%$ ). SSS alone (AUC, 0.706) had differentiation similar to stress MBF, uncorrected MFR, and corrected MFR. The addition of abnormal regional perfusion to stress MBF, corrected MFR, and uncorrected MFR did not result in a statistically significant improvement in discrimination of patients with CAV compared with quantitative markers alone
(Supplemental Fig. 1; supplemental materials are available at http:// jnm.snmjournals.org).

\section{Prognostic Utility of ${ }^{82} \mathrm{Rb}$ PET}

Kaplan-Meier survival curves for semiquantitative regional analysis are shown in Figure 2. An SSS of at least 4 (log-rank $P=0.017)$ was associated with increased all-cause mortality. Abnormal SRS or SDS was not associated with all-cause mortality. Kaplan-Meier survival curves for quantitative PET variables are shown in Figure 3. Abnormal MFR (log-rank $P<0.001$ ), corrected MFR (log-rank $P<0.001$ ), and stress MBF (log-rank $P=0.002)$ were associated with increased all-cause mortality.

In a univariable Cox proportional hazards analysis (Table 3), stress MBF (unadjusted hazard ratio [HR], 0.56; 95\% confidence interval $[\mathrm{CI}], 0.35-0.90 ; P=0.017)$, uncorrected MFR (unadjusted HR, 0.34; 95\% CI, 0.19-0.62; $P<0.001$ ), and corrected MFR (unadjusted HR, 0.44; 95\% CI, 0.26-0.74; $P=0.002$ ) were associated with all-cause mortality. A higher SSS was also associated with increased all-cause mortality (unadjusted HR, 1.09; 95\% CI, 1.03-1.15; $P=0.002$ ), as were

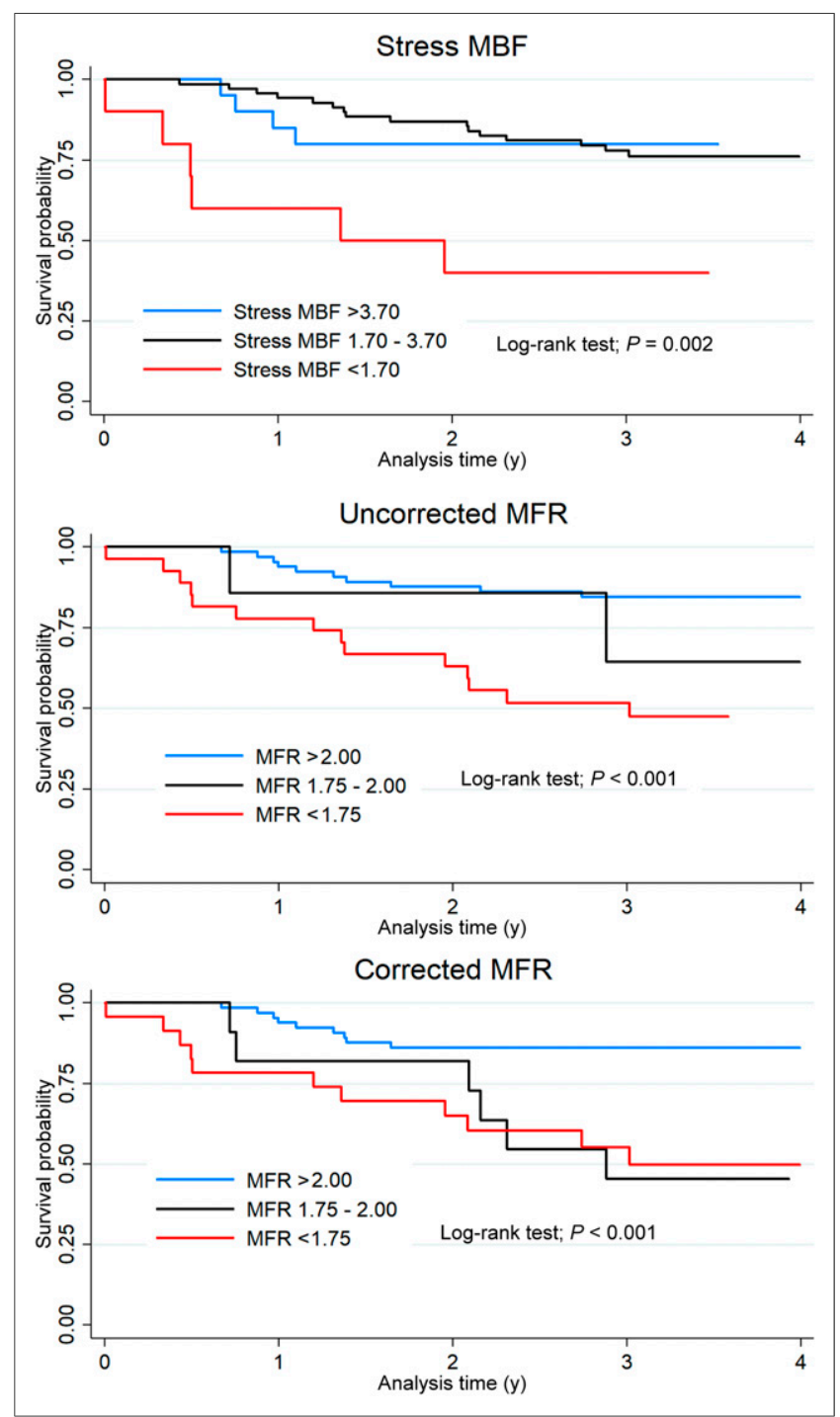

FIGURE 3. Kaplan-Meier survival curves for all-cause mortality stratified by quantitative PET results. 
TABLE 3

Univariable and Multivariable Association with All-Cause Mortality

\begin{tabular}{|c|c|c|c|c|}
\hline Variable & Unadjusted HR & $P$ & Adjusted HR & $P$ \\
\hline Age & $1.08(1.03-1.14)$ & $<0.001$ & $1.10(1.04-1.17)$ & 0.001 \\
\hline Male & $1.37(0.52-3.65)$ & 0.523 & - & - \\
\hline Body mass index & $0.97(0.80-1.05)$ & 0.442 & - & - \\
\hline LVEF & $0.95(0.92-0.98)$ & 0.001 & $0.98(0.94-1.02)$ & 0.232 \\
\hline \multicolumn{5}{|l|}{ Cardiac risk factors } \\
\hline Hypertension & $0.54(0.23-1.28)$ & 0.161 & - & - \\
\hline Diabetes & $1.83(0.84-3.99)$ & 0.129 & - & - \\
\hline Dyslipidemia & $1.55(0.58-.11)$ & 0.380 & - & - \\
\hline Renal failure & $1.68(0.58-4.88)$ & 0.339 & - & - \\
\hline Cytomegalovirus viremia & $0.77(0.23-2.55)$ & 0.666 & - & - \\
\hline Acute cellular rejection & $1.43(0.54-3.79)$ & 0.474 & - & - \\
\hline Antibody-mediated rejection & $1.88(0.84-4.20)$ & 0.124 & - & - \\
\hline \multicolumn{5}{|l|}{ PET parameters } \\
\hline SRS & $1.15(1.01-1.31)$ & 0.033 & $0.71(0.20-2.54)$ & 0.602 \\
\hline SSS & $1.09(1.03-1.15)$ & 0.002 & $1.02(0.29-3.54)$ & 0.976 \\
\hline SDS & $1.15(1.06-1.26)$ & 0.001 & $1.22(0.35-4.19)$ & 0.754 \\
\hline Rest MBF & $1.81(0.78-4.19)$ & 0.166 & - & - \\
\hline Stress MBF & $0.56(0.35-0.90)$ & 0.017 & $1.14(0.64-2.05)$ & 0.656 \\
\hline Uncorrected MFR ${ }^{\star}$ & $0.34(0.19-0.62)$ & $<0.001$ & $0.30(0.11-0.81)$ & 0.017 \\
\hline Corrected MFR* & $0.44(0.26-0.74)$ & 0.002 & $0.43(0.20-0.90)$ & 0.025 \\
\hline
\end{tabular}

*Multivariable analysis was performed separately with corrected and uncorrected MFR.

Data in parentheses are $95 \%$ Cls.

age and lower LVEF. However, in a multivariable model, only age and MFR continued to be associated with all-cause mortality (uncorrected: adjusted HR, $0.30 ; P=0.017$; corrected: adjusted HR, $0.43 ; P=0.025)$. The addition of uncorrected MFR significantly improved death prediction when added to age, LVEF, SRS, SSS, SDS, and stress $\operatorname{MBF}\left(\chi^{2}, 6.5 ; P=0.011\right)$, with less improvement by adding corrected MFR $\left(\chi^{2}, 5.4 ; P=0.020\right)$.

Receiver-operating-characteristic curves for prediction of all-cause mortality are shown in Figure 4. Uncorrected MFR showed improved discrimination for all-cause mortality compared with stress $\mathrm{MBF}$ (AUC, 0.748 vs. $0.639 ; P=0.048$ ). However, there was no significant difference in mortality prediction between MFR and corrected MFR. Prediction of all-cause mortality with SSS alone (AUC, 0.593) was significantly worse than with $\operatorname{MFR}(P=0.007)$ or corrected MFR $(P=0.019)$, but not stress $\operatorname{MBF}(P=0.435)$. The addition of abnormal regional perfusion to MFR or stress MBF analyses did not significantly improve the prognostic accuracy compared with quantitative markers alone (Supplemental Fig. 2).

\section{Cardiovascular Mortality}

Cardiovascular mortality occurred in 16 (16.2\%) patients. Univariable and multivariable Cox proportional hazards analyses of the association with cardiovascular mortality are shown in Supplemental Table 1. Stress MBF was associated with increased cardiovascular mortality in univariable analysis (unadjusted HR, $0.37 ; P=0.002$ ) but not multivariable analysis (adjusted HR, 1.08; $P=0.848$ ). Uncorrected MFR was associated with increased cardiovascular mortality in univariable analysis (unadjusted HR, 0.17;
$P<0.001$ ) and multivariable analysis (adjusted HR, 0.05; $P=$ 0.001). Receiver-operating-characteristic curves for prediction of cardiovascular mortality are shown in Supplemental Figure 3.

\section{Comparison of Previously Described Thresholds}

Table 4 summarizes the diagnostic and prognostic utility of previously described abnormal thresholds and their combinations. Uncorrected MFR was used because discrimination was numerically superior to corrected MFR in our prior analyses. With respect to diagnosis of $\mathrm{CAV}$, stress $\mathrm{MBF}$ performed well among individual variables, but with restrictive thresholds. A stress MBF of less than 3.7 had the highest sensitivity but classified $79.8 \%$ of patients as abnormal, whereas a stress MBF of less than 1.7 had the highest specificity, with only $10.1 \%$ of patients classified as abnormal. The highest overall accuracy was achieved with an MFR of less than 2.0 or the combined marker of the presence of any SSS of at least 4 , LVEF of $45 \%$ or less, or MFR of less than 1.75. Regarding prognosis, a stress MBF of less than 1.7 was the single predictor that identified patients with the highest risk annualized mortality rate $(35.8 \%)$. Patients with reduced stress $\operatorname{MBF}(<1.7)$ and either an SSS of more than 1 or an LVEF of $45 \%$ or less had an annual mortality rate of $60.7 \%$. Patients with preserved MFR $(\geq 2.0)$ were at the lowest risk (annual mortality rate, $4.7 \%$ ).

\section{DISCUSSION}

PET MPI with MBF quantification has been shown to improve the diagnosis of $\mathrm{CAV}$ and provide incremental prognostic value in 


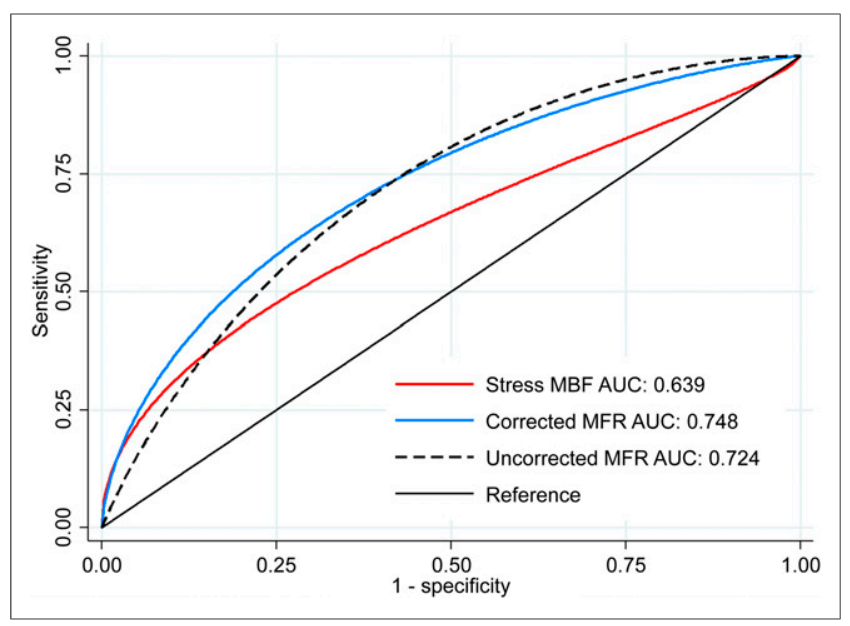

FIGURE 4. Receiver-operating-characteristic curves for identifying allcause mortality during follow-up. Uncorrected MFR AUC was significantly larger than stress MBF AUC $(P=0.047)$. There was no significant difference between uncorrected MFR AUC and corrected MFR AUC $(P=0.681)$.

patients after heart transplantation. However, there is no consensus on which marker, stress MBF or MFR, should be applied for either diagnostic or prognostic purposes (21). In this study, we demonstrated that stress MBF, uncorrected MFR, and corrected MFR were equivalent in discriminating patients with significant CAV. For prognosis, we found that reduced MFR offered superior discrimination for all-cause mortality, compared with stress MBF. Finally, we found that correcting for rate-pressure product did not improve the prognostic or diagnostic accuracy of MFR.

Our study adds to a growing body of literature describing the diagnostic utility of quantitative PET analysis. Bravo et al. reported that in patients imaged by ${ }^{13} \mathrm{~N}-\mathrm{NH}_{3}$ PET MPI, stress MBF and LVEF combined with regional perfusion improved detection of significant $\mathrm{CAV}$, with an AUC of 0.88 compared with 0.82 (10). Konerman et al. reported that MFR or stress MBF numerically, but not significantly, improved discrimination of CAV compared with regional perfusion when assessed by ${ }^{82} \mathrm{Rb}$ PET ( 8 ). Chih et al. found that stress MBF and MFR had similar discrimination of CAV when defined angiographically or by intravascular ultrasound (22). In our population, stress MBF, uncorrected MFR, and corrected MFR had comparable performance for diagnosis of significant CAV. These results suggest that either stress MBF or MFR may improve diagnosis of CAV and that if differences in discrimination are present, they are likely not clinically significant.

Our study also confirmed the prognostic utility of PET MPI in patients after heart transplantation. Mc Ardle et al. followed 140 cardiac transplant patients who underwent ${ }^{82} \mathrm{Rb}$ PET for a median 18.2 mo of follow-up, during which 14 events occurred, including 9 deaths (9). They demonstrated that MFR, but not stress MBF, was associated with increased adverse events, with an MFR of less than 1.5 conferring a 4-fold increase in risk. Konerman et al. (8) reported similar associations with a combined outcome of death $(n=2)$, acute coronary syndrome $(n=5)$, revascularization $(n=8)$, or heart failure $(n=15)$. Both studies used uncorrected MFR. We expanded on this evidence by including a greater number of hard events and observed that only reduced MFR was associated with increased all-cause mortality in adjusted analyses. Additionally, the discriminatory value of MFR was superior to that of stress MBF for identifying patients who died during follow-up. Serial evaluation with PET has been shown to further refine prognostication (23). Our findings provide evidence that quantitative PET blood flow analysis, particularly with MFR, has significant prognostic utility after heart transplantation.

Lastly, we describe the performance of previously described abnormal thresholds in our cohort. Although semiquantitative perfusion abnormalities were associated with increased all-cause mortality, they did not significantly improve diagnosis of CAV or prognostication when added to stress MBF or MFR. Further, we found that preserved MFR (defined as $\geq 2.0$ ) identified a group of patients with the lowest rate of all-cause mortality during follow-up. Combining reduced LVEF with reduced MFR (or MBF) identified a group of patients with annual mortality over $50 \%$. Additional patients at high mortality risk were identified by combining the presence of severely reduced stress MBF with abnormal regional perfusion or reduced LVEF. As a

TABLE 4

Diagnostic and Prognostic Values of Previously Reported Thresholds

\begin{tabular}{|c|c|c|c|c|c|}
\hline \multirow[b]{2}{*}{ Cutoff } & \multirow[b]{2}{*}{ Abnormal $(n)$} & \multicolumn{2}{|c|}{ Diagnosis of CAV grade $2 / 3$} & \multicolumn{2}{|c|}{$\begin{array}{c}\text { Annualized all-cause } \\
\text { mortality rate }\end{array}$} \\
\hline & & Sensitivity & Specificity & Abnormal & Normal \\
\hline MFR $<2.0$ & $34(34.3 \%)$ & $71.4 \%$ & $71.8 \%$ & $17.7 \%$ & $4.7 \%$ \\
\hline MFR $<1.75$ & $27(27.3 \%)$ & $57.1 \%$ & $77.7 \%$ & $19.6 \%$ & $5.2 \%$ \\
\hline Stress MBF $<3.7$ & $79(79.8 \%)$ & $92.9 \%$ & $22.4 \%$ & $9.0 \%$ & $6.7 \%$ \\
\hline Stress MBF $<1.7$ & $10(10.1 \%)$ & $42.9 \%$ & $95.3 \%$ & $35.8 \%$ & $7.0 \%$ \\
\hline SSS $>1$ & $32(32.2 \%)$ & $64.3 \%$ & $72.9 \%$ & $12.3 \%$ & $7.0 \%$ \\
\hline SSS $>3$ & $15(15.2 \%)$ & $64.3 \%$ & $92.9 \%$ & $18.7 \%$ & $7.1 \%$ \\
\hline LVEF $\leq 45$ & $10(10.1 \%)$ & $42.9 \%$ & $95.3 \%$ & $25.0 \%$ & $7.2 \%$ \\
\hline $\mathrm{MBF}<1.7$ and $\mathrm{SSS}>1$ or $\mathrm{LVEF} \leq 45$ & $8(8.1 \%)$ & $42.9 \%$ & $97.7 \%$ & $60.7 \%$ & $6.8 \%$ \\
\hline SSS $\geq 4$, LVEF $\leq 45$ or MFR $<1.75$ & $36(36.4 \%)$ & $71.4 \%$ & $69.4 \%$ & $15.4 \%$ & $5.0 \%$ \\
\hline LVEF $\leq 45 \%$ and MFR $<1.75^{\star}$ & $5(5.1 \%)$ & $35.7 \%$ & $100.0 \%$ & $51.6 \%$ & $7.4 \%$ \\
\hline
\end{tabular}

*Same patients would be identified using MBF $<1.7$ and LVEF $<45 \%$.

Uncorrected MFR was used because it was numerically superior in all models. 
point of reference, the International Society of Heart and Lung Transplantation guidelines suggest that patients with end-stage heart failure and estimated 1-y mortality over $20 \%$ be considered for transplant listing (24). Therefore, PET may have a role for identifying patients who may benefit from retransplantation before they develop significant heart failure symptoms or recurrent hospitalizations. Physicians should integrate multiple parameters to improve diagnostic sensitivity or risk stratification based on individual clinical scenarios.

Our study had a few important limitations. This was a retrospective study of patients from a single center. Our sample size was small, but it was comparable to prior studies. The patients who were referred for PET represented a high-risk cohort as evidenced by the high annual mortality rates. PET perfusion studies were performed as part of routine clinical practice; therefore, the interval between invasive coronary angiography and PET scanning was not standardized and the delay had been over $2 \mathrm{y}$ in one quarter of patients. Although this delay may have affected the assessment of diagnostic accuracy, most patients had received their transplant more than $10 \mathrm{y}$ previously and therefore were at a stage at which CAV tends to progress less rapidly (2). Our use of a software package different from that in other published studies may explain some of the variation in findings. However, the correlation across software packages is excellent (25). Additionally, our results confirm that PET has diagnostic and prognostic utility regardless of the software package used.

\section{CONCLUSION}

We confirmed the diagnostic and prognostic utility of PET flow quantitation in posttransplant patients. Stress MBF and MFR had similar diagnostic utility, and correcting for rate-pressure product did not improve diagnostic or prognostic accuracy. However, we found that uncorrected MFR was superior to stress MBF for prognostication. Preserved MFR identifies low-risk patients, whereas the presence of multiple abnormal parameters identifies patients at the highest risk.

\section{DISCLOSURE}

This work was supported in part by the Dr. Miriam and Sheldon Adelson Medical Research Foundation. Robert Miller receives funding support from the Arthur J.E. Child Fellowship grant. Daniel Berman and Piotr Slomka participate in software royalties for QPS software at Cedars-Sinai Medical Center. No other potential conflict of interest relevant to this article was reported.

\section{KEY POINTS}

QUESTION: What is the diagnostic and prognostic utility of MBF and MFR in patients after cardiac transplantation?

PERTINENT FINDINGS: We specifically compared the performance of MFR with and without correction for rate-pressure product and found that uncorrected values have numerically higher diagnostic and prognostic utility. Additionally, we showed that stress MBF and MFR have similar diagnostic utility, whereas uncorrected MFR has superior prediction of all-cause mortality.

IMPLICATIONS FOR PATIENT CARE: Physicians should integrate multiple parameters to improve diagnostic sensitivity or risk stratification based on individual clinical scenarios.

\section{REFERENCES}

1. Lund LH, Khush KK, Cherikh WS, et al. The Registry of the International Society for Heart and Lung Transplantation: thirty-fourth adult heart transplantation report-2017; focus theme: allograft ischemic time. J Heart Lung Transplant. 2017; 36:1037-1046.

2. Pollack A, Nazif T, Mancini D, Weisz G. Detection and imaging of cardiac allograft vasculopathy. JACC Cardiovasc Imaging. 2013;6:613-623.

3. Lund LH, Edwards LB, Kucheryavaya AY, et al. The Registry of the International Society for Heart and Lung Transplantation: thirty-first official adult heart transplant report. J Heart Lung Transplant. 2014;33:996-1008.

4. Stone GW, Maehara A, Lansky AJ, et al. A prospective natural-history study of coronary atherosclerosis. N Engl J Med. 2011;364:226-235.

5. Chirakarnjanakorn S, Starling RC, Popovic ZB, Griffin BP, Desai MY. Dobutamine stress echocardiography during follow-up surveillance in heart transplant patients: diagnostic accuracy and predictors of outcomes. J Heart Lung Transplant. 2015;34:710-717.

6. Thompson D, Koster MJ, Wagner RH, Heroux A, Barron JT. Single photon emission computed tomography myocardial perfusion imaging to detect cardiac allograft vasculopathy. Eur Heart J Cardiovasc Imaging. 2012;13:271-275.

7. Bravo PE, Di Carli MF, Dorbala S. Role of PET to evaluate coronary microvascular dysfunction in non-ischemic cardiomyopathies. Heart Fail Rev. 2017;22: 455-464.

8. Konerman MC, Lazarus JJ, Weinberg RL, et al. Reduced myocardial flow reserve by positron emission tomography predicts cardiovascular events after cardiac transplantation. Circ Heart Fail. 2018;11:e004473.

9. Mc Ardle BA, Davies RA, Chen L, et al. Prognostic value of rubidium-82 positron emission tomography in patients after heart transplant. Circ Cardiovasc Imaging. 2014;7:930-937

10. Bravo PE, Bergmark BA, Vita T, et al. Diagnostic and prognostic value of myocardial blood flow quantification as non-invasive indicator of cardiac allograft vasculopathy. Eur Heart J. 2018;39:316-323.

11. Mehra MR, Crespo-Leiro MG, Dipchand A, et al. International Society for Heart and Lung Transplantation working formulation of a standardized nomenclature for cardiac allograft vasculopathy: 2010. J Heart Lung Transplant. 2010;29: 717-727.

12. Slomka PJ, Diaz-Zamudio M, Dey D, et al. Automatic registration of misaligned $\mathrm{CT}$ attenuation correction maps in Rb-82 PET/CT improves detection of angiographically significant coronary artery disease. J Nucl Cardiol. 2015;22:12851295.

13. Henzlova MJ, Duvall WL, Einstein AJ, Travin MI, Verberne HJ. ASNC imaging guidelines for SPECT nuclear cardiology procedures: stress, protocols, and tracers. J Nucl Cardiol. 2016;23:606-639.

14. Dekemp RA, Declerck J, Klein R, et al. Multisoftware reproducibility study of stress and rest myocardial blood flow assessed with 3D dynamic PET/CT and a 1-tissue-compartment model of ${ }^{82} \mathrm{Rb}$ kinetics. J Nucl Med. 2013;54:571-577.

15. Chow BJ, Ananthasubramaniam K, deKemp RA, Dalipaj MM, Beanlands RS, Ruddy TD. Comparison of treadmill exercise versus dipyridamole stress with myocardial perfusion imaging using rubidium- 82 positron emission tomography. J Am Coll Cardiol. 2005;45:1227-1234.

16. Nakazato R, Berman DS, Dey D, et al. Automated quantitative Rb-82 3D PET/ CT myocardial perfusion imaging: normal limits and correlation with invasive coronary angiography. J Nucl Cardiol. 2012;19:265-276.

17. Lortie M, Beanlands RS, Yoshinaga K, Klein R, Dasilva JN, DeKemp RA. Quantification of myocardial blood flow with ${ }^{82} \mathrm{Rb}$ dynamic PET imaging. Eur J Nucl Med Mol Imaging. 2007;34:1765-1774.

18. Youden WJ. Index for rating diagnostic tests. Cancer. 1950;3:32-35.

19. Hicks KA, Mahaffey KW, Mehran R, et al. 2017 cardiovascular and stroke endpoint definitions for clinical trials. Circulation. 2018;137:961-972.

20. DeLong ER, DeLong DM, Clarke-Pearson DL. Comparing the areas under two or more correlated receiver operating characteristic curves: a nonparametric approach. Biometrics. 1988;44:837-845.

21. Miller RJH, Kobashigawa JA, Berman DS. Should positron emission tomography be the standard of care for non-invasive surveillance following cardiac transplantation? J Nucl Cardiol. 2019;26:655-659.

22. Chih S, Chong AY, Erthal F, et al. PET assessment of epicardial intimal disease and microvascular dysfunction in cardiac allograft vasculopathy. J Am Coll Cardiol. 2018;71:1444-1456.

23. Feher A, Srivastava A, Quail MA, et al. Serial assessment of coronary flow reserve by rubidium- 82 positron emission tomography predicts mortality in heart transplant recipients. JACC Cardiovasc Imaging. October 12, 2018 [Epub ahead of print].

24. Mehra MR, Canter CE, Hannan MM, et al. The 2016 International Society for Heart Lung Transplantation listing criteria for heart transplantation: a 10-year update. J Heart Lung Transplant. 2016;35:1-23.

25. Nesterov SV, Deshayes E, Sciagrà R, et al. Quantification of myocardial blood flow in absolute terms using ${ }^{82} \mathrm{Rb}$ PET imaging: the RUBY-10 Study. JACC Cardiovasc Imaging. 2014;7:1119-1127. 